\title{
Postictal switch in blood flow distribution and temporal lobe seizures
}

\author{
Mark R Newton, Samuel F Berkovic, Mark C Austin, Christopher C Rowe, \\ W John McKay, Peter F Bladin
}

\begin{abstract}
The ictal increase of regional cerebral blood flow has yet to be fully utilised in the investigation of focal seizures. Although single photon emission tomography (SPECT) is being increasingly used in the localisation of epileptic foci, the evolution and time courses of the peri-ictal perfusion changes have yet to be clarified. We performed serial SPECT studies in the interictal, ictal and immediate postictal states in 12 patients with refractory temporal lobe epilepsy to define the patterns and duration of peri-ictal cerebral blood flow changes. Visual analysis showed a constant pattern of unilateral global increases in temporal lobe perfusion during seizures which suddenly switched to a pattern of relative mesial temporal (hippocampal) hyperperfusion and lateral temporal hypoperfusion in the immediate postictal period. Quantitative analysis confirmed the visual assessment. Lateral temporal cortex ictal/normal side to side ratios were increased by mean $35.1 \%$ (95\% confidence interval $21 \cdot 8 \%$ to $48 \cdot 4 \%$ ) more in the ictal studies than in the interictal studies and mesial temporal cortex ratios increased by mean $30.8 \%(22.4 \%$ to $39 \cdot 2 \%)$. In the postictal state, however, lateral temporal ratios were reduced by mean $7 \cdot 7 \%(-15 \cdot 8 \%$ to $0 \cdot 4 \%)$ compared with interictal values, whereas mesial temporal perfusion was maintained compared with the interictal studies. These observations provide critical information for interpreting scans which can be used in the localisation of epileptic foci. This postictal switch in blood flow patterns may reflect the underlying metabolic processes of neuronal activation and recovery and have implications for understanding the neurobiology of human epileptic seizures.
\end{abstract}

(F Neurol Neurosurg Psychiatry 1992;55:891-894)

The abnormal synchronised discharge of a neuronal aggregate that characterises focal epileptic seizures is accompanied by focal hyperperfusion $^{1-5}$ and hypermetabolism. ${ }^{67}$ Functional imaging with positron emission tomography (PET) and single photon emission computed tomography (SPECT) has shown a variety of peri-ictal blood flow patterns, ${ }^{58-13}$ presumably reflecting the rapid changes of blood flow accompanying focal seizures. Peri- ictal studies with PET can be performed only serendipitously when seizures happen to occur during the scan. SPECT radionuclides such as ${ }^{99 \mathrm{~m}}$ Technetium-hexamethyl propyleneamineoxime (HMPAO) rapidly cross the bloodbrain barrier and reside in cerebral tissue for several hours. Isotope can thus be injected during an observed seizure and the patient can then be scanned within the next few hours, with the scan reflecting cerebral blood flow (CBF) at the time of injection. ${ }^{14}$ These properties permit the prospective and serial evaluation of changes in CBF associated with focal seizures, which typically last less than two minutes.

We previously reported the early postictal perfusion changes with SPECT, which allowed accurate prediction of seizure focus in $69 \%$ of a series of patients with refractory temporal lobe epilepsy. ${ }^{11-13}$ We have since sought to determine the diagnostic value of ictal and postictal SPECT scans by examining a series of ictal and postictal paired studies in the same patients with qualitative and quantitative image analysis. We found a remarkable difference in the ictal and postictal perfusion patterns, representing a postictal switch in blood flow distribution. This finding is important for clinical diagnosis of seizure localisation and for understanding the pathophysiology of temporal lobe epilepsy.

\section{Methods}

Twelve patients (mean age 28, (range 16-46) years; seven women) with intractable unilateral temporal lobe epilepsy diagnosed on clinical and EEG criteria, and MRI ${ }^{15}$ and neuropsychological data were studied (table 1). Periictal SPECT is a routine investigation for prospective therapeutic temporal lobectomy in our centre, ${ }^{11-13}$ and the 12 patients analysed were selected because they had undergone paired SPECT studies for both ictal and postictal states.

An interictal study was performed when the patient had been seizure free for at least 24 hours, with full medication. The ${ }^{99} \mathrm{Tc}$ HMPAO was injected with the patient in the resting state (supine, eyes closed, and silent with a minimum of background noise). Patients were continuously monitored with video recording and EEG for periods of up to two weeks (with reduced medication) and underwent ${ }^{99 m}$ Tc-HMPAO injection as soon as possible after onset of the seizure. SPECT images derived from injections during or up to 30 seconds after the end of a seizure were 
Table 1 Localisation data in 12 patients studied with ictal and postictal SPECT *

\begin{tabular}{|c|c|c|c|c|c|c|}
\hline Patient & Ictal $E E G$ & $\begin{array}{l}\text { Interictal } \\
\text { SPECT }\end{array}$ & $\begin{array}{l}\text { Ictal } \\
\text { SPECT }\end{array}$ & $\begin{array}{l}\text { Postictal } \\
\text { SPECT }\end{array}$ & $M R I \dagger$ & Pathology \\
\hline $\begin{array}{r}1 \\
2 \\
3 \\
4 \\
5 \\
6 \\
7 \\
8 \\
9 \\
10 \\
11 \\
12\end{array}$ & 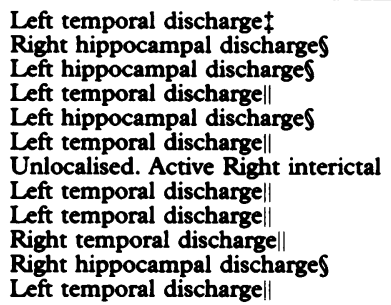 & $\begin{array}{l}\text { Not lateralised } \\
\text { Right } \\
\text { Left } \\
\text { Not lateralised } \\
\text { Left } \\
\text { Left } \\
\text { Not lateralised } \\
\text { Left } \\
\text { Left } \\
\text { Not lateralised } \\
\text { Right } \\
\text { Left }\end{array}$ & $\begin{array}{l}\text { Left } \\
\text { Right } \\
\text { Left } \\
\text { Left } \\
\text { Left } \\
\text { Left } \\
\text { Right } \\
\text { Left } \\
\text { Left } \\
\text { Right } \\
\text { Right } \\
\text { Left }\end{array}$ & $\begin{array}{l}\text { Not lateralised } \\
\text { Right } \\
\text { Left } \\
\text { Left } \\
\text { Left } \\
\text { Left } \\
\text { Right } \\
\text { Left } \\
\text { Not lateralised } \\
\text { Right } \\
\text { Not lateralised } \\
\text { Left }\end{array}$ & $\begin{array}{l}\text { Left HS } \\
\text { Right HS } \\
\text { Left HS } \\
\text { Left tumour } \\
\text { Normal } \\
\text { Left HS } \\
\text { Right HS } \\
\text { Left dysplasia } \\
\text { Left HS } \\
\text { Right HS } \\
\text { Normal } \\
\text { Left HS }\end{array}$ & $\begin{array}{l}\text { HS } \\
\text { HS } \\
\text { HS } \\
\text { Ganglioglioma } \\
\text { Normal } \\
\text { HS } \\
\text { HS } \\
\text { Dysplasia } \\
\text { HS } \\
\text { Cortical gliosis } \\
\text { Normal } \\
\text { HS }\end{array}$ \\
\hline
\end{tabular}

HS = hippocampal sclerosis.

^SPECT results derived from blinded visual assessment. Interictal SPECT localised by focal hypoperfusion, ictal SPECT by focal hyperperfusion, and postictal SPECT by mesial hyerperfusion with lateral hypoperfusion.

tMRI diagnosis of hippocampal sclerosis was made as previously described and validated. ${ }^{15}$

tSurface EEG.

SDepth EEG.

Sphenoidal EEG.

ๆHippocampal tissue not found on pathology specimen.

called "ictal studies", as the appearances of scans in all cases were consistently similar. All 12 patients underwent an additional injection on another occasion more than 30 seconds after seizure end (postictal study; mean (SD) delay $1.46(0.4) \mathrm{min})$. Exact timing of the injections was made possible by the timer linked to the video and EEG, which was read on playback of all seizures. The paired seizures studied were of a stereotyped pattern and similar length for each patient. Patients were scanned within two hours of injection, with a Starcam 400AC gammacamera (General Electric, Milwaukee, USA). Data acquisition, reconstruction, and display methods have been described previously. ${ }^{12}$

Scans were qualitatively interpreted by three blinded observers. The blood flow changes were quantitated by determining a ratio of counts, dividing the number in the epileptogenic side by that in the normal side, in the following regions of interest; mesial, lateral, and posterior temporal regions; lateral frontal and parietal cortices; basal ganglia; and cerebellar hemispheres. Three contiguous $3.2 \mathrm{~mm}$ transverse slices were summed and regions of interest were drawn using rectangular boxes. The volume studied was $4.9 \mathrm{~cm}^{3}(5 \times 10 \times 3$ pixels) for all regions except for the cerebellum $\left(7 \times 7 \times 3\right.$ pixels, $\left.4.8 \mathrm{~cm}^{3}\right)$ and basal ganglia $\left(5 \times 5 \times 3\right.$ pixels, $\left.2.5 \mathrm{~cm}^{3}\right)$. Normal data were obtained from scans of 18 healthy male volunteers where $L / R$ ratios were calculated for the same regions of interest. Mean ratios for each region of interest in the ictal, postictal, and interictal patient studies were compared with each other by using paired $t$ tests and with control values by using multiple unpaired $t$ tests. Conversion of ratios to logarithms was unnecessary as ratios varied beween 0.7 and $1 \cdot 7$.

\section{Results}

All patients were studied with ictal and postictal SPECT; the length of seizures was similar in each patient for the two studies (ictal, mean length $1 \mathrm{~min} 33 \mathrm{~s}$, postictal, mean length $1 \mathrm{~min} 25 \mathrm{~s}$ ). Ictal images in all 12 patients showed a pronounced global increase in temporal lobe blood flow on the epileptogenic side (figure). This pattern was seen when injections were given during clinical and EEG ictal activity or up to 30 seconds after its cessation and was easily identified in all 12 cases by the three blinded observers independently (table 1). Postictal studies showed a switch in distribution of blood flow with hypoperfusion of the epileptogenic lobe and hemisphere in all 12 studies and residual hyperperfusion of the mesial temporal region in seven. The postictal changes were not always striking. In nine patients they were obvious to all three observers but in the three others they were not sufficiently clear for blinded lateralisation independent of other data.

Hypoperfusion of the frontal and parietal cortex was seen ipsilateral to the discharging focus in the ictal studies and persisted into the postictal period. One patient progressed to hemigeneralisation after his complex partial seizure. SPECT showed increased perfusion in the contralateral motor cortex in this case, in addition to the temporal lobe hyperperfusion.

Perfusion ratios for all regions of interest in the patients' interictal state did not differ from those in the control group, except for the mesial temporal region where there was a significant decrease on the side of the epileptic focus (table 2). Quantitative analysis of the ictal and postictal studies confirmed the qualitative interpretation of the images (table 3 ).

Table 2 Side to side perfusion ratios in the interictal state, * Values are means (SD)

\begin{tabular}{lrrl}
\hline $\begin{array}{l}\text { Region of } \\
\text { Interest }\end{array}$ & Controls & \multicolumn{1}{c}{ Patients } & $p$ Value \\
\hline Lateral temporal & $98(2.5)$ & $100(4 \cdot 39)$ & NS \\
Mesial temporal & $101(1.9)$ & $94(6.24)$ & $<0.05$ \\
Posterior temporal & $101(2.5)$ & $100(3.04)$ & NS \\
Lateral frontal & $99(2.3)$ & $99(3.31)$ & NS \\
Parietal & $99(1.7)$ & $101(3.92)$ & NS \\
Cerebellum & $100(1.9)$ & $99(3.75)$ & NS \\
Basal ganglia & $99(2.1)$ & $100(2.0)$ & NS \\
\hline
\end{tabular}

* Side to side ratios (multiplied by 100) calculated from left or right side counts for controls, and ictal or normal side for patients, were compared in each region of interest by using unpaired $t$ tests. 


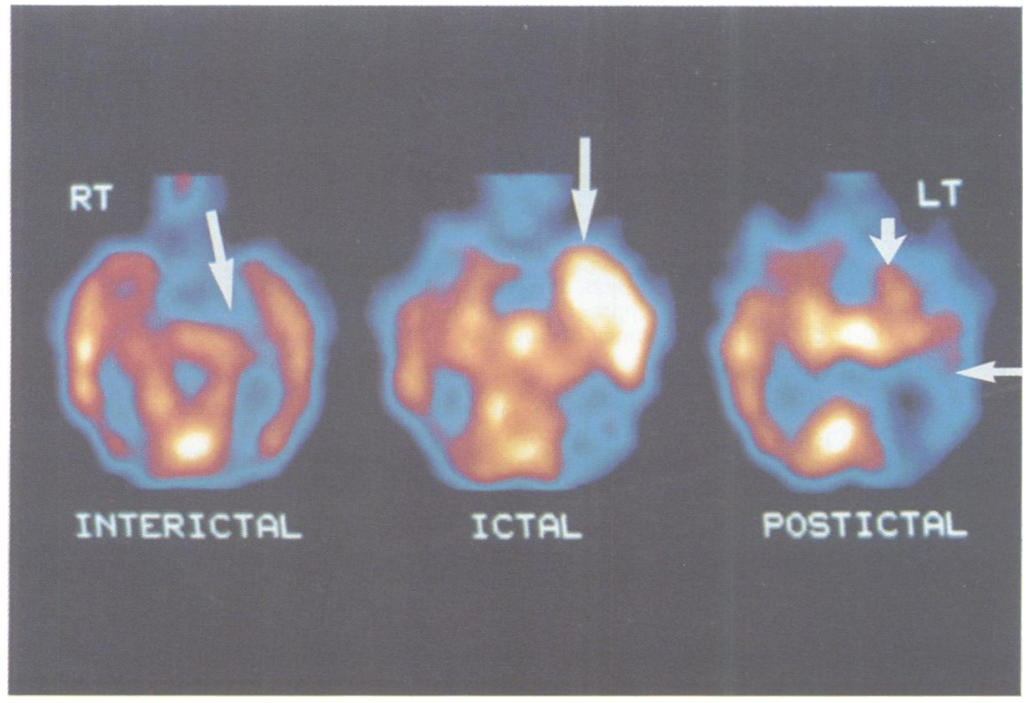

Transverse images through the temporal lobes for interictal, ictal, and postictal studies of patient 6 (left temporal lobe seizures). Colour scale from white to red to blue represents a spectrum of high to low counts. In the interictal study there is a small region of hypoperfusion in the left mesial temporal lobe (arrow). The ictal study shows a pronounced global increase in temporal perfusion (large arrow). The 1.5 minute postictal study shows lateral temporal hypoperfusion (horizontal arrow) with residual mesial hyperperfusion (vertical arrow) on the left.

The lateral temporal, mesial temporal, and basal ganglia ratios were significantly increased in the ictal studies in comparison to the interictal, postictal and control data. The increase over interictal values was $35 \cdot 1 \%(95 \%$ confidence interval $21 \cdot 8 \%$ to $48 \cdot 4 \%$ ) ( $p<$ 0.0005 ) for the lateral temporal area and $30.8 \%(22.4 \%$ to $39.2 \%)(p<0.0001)$ for the mesial temporal region. Comparison of the postictal to the interictal scans showed that the postictal mesial temporal ratios were unchanged, whereas the posterior and lateral temporal ratios were reduced (although the lateral temporal change just failed to reach significance). Frontal and parietal ratios were significantly decreased in the postictal state, confirming the visual interpretation of the scans.

No discrepancies were found between SPECT and EEG with regard to side of ictal activity. In patient 7, ictal EEG data were not useful in localizing the seizure focus, but because clinical, interictal EEG, SPECT, MRI, and neuropsychological data concurred on the localisation, a temporal lobectomy was performed; pathological examination con-

Table 3 Relative regional cerebral blood flow changes in the ictal and postictal states compared to the interictal condition *

\begin{tabular}{|c|c|c|c|c|}
\hline \multirow[b]{2}{*}{$\begin{array}{l}\text { Region of } \\
\text { interest }\end{array}$} & \multicolumn{2}{|l|}{ Ictal studies } & \multicolumn{2}{|l|}{ Postictal studies } \\
\hline & $\begin{array}{l}\text { Mean \% change } \\
\text { (95\% confidence } \\
\text { interval) }\end{array}$ & p Value & $\begin{array}{l}\text { Mean \% change } \\
\text { (95\% confidence } \\
\text { interval) }\end{array}$ & p Value \\
\hline $\begin{array}{l}\text { Lateral temporal } \\
\text { Mesial temporal } \\
\text { Posterior temporal } \\
\text { Lateral frontal } \\
\text { Parietal } \\
\text { Basal ganglia } \\
\text { Cerebellum }\end{array}$ & $\begin{array}{c}35.1(21.8 \text { to } 48.4) \\
30.8(22.4 \text { to } 39.2) \\
11.1(-3.5 \text { to } 25 \cdot 7) \\
-5.7(-13.9 \text { to } 2.5) \\
1.2(-7.5 \text { to } 9.9) \\
9.6(2.7 \text { to } 16.5) \\
-2.3(-5.2 \text { to } 0.6)\end{array}$ & $\begin{array}{l}<0.0005 \\
<0.0001 \\
\text { NS } \\
\text { NS } \\
\text { NS } \\
<0.02 \\
\text { NS }\end{array}$ & $\begin{array}{l}-7.7(-15.8 \text { to } 0.4) \\
2.0(-6.3 \text { to } 10.3) \\
-8.5(-14.0 \text { to }-3.0) \\
-12.3(-17.6 \text { to }-7.0) \\
-7.7(-13.9 \text { to }-1.5) \\
-0.2(-4.3 \text { to } 3.9) \\
0.6(-1.7 \text { to } 2.9)\end{array}$ & $\begin{array}{l}0.058 \\
\text { NS } \\
<0.01 \\
<0.0005 \\
<0.05 \\
\text { NS } \\
\text { NS }\end{array}$ \\
\hline
\end{tabular}

*Percentage differences in mean ratio changes (epileptic side/normal side) from interictal to ictal and from interictal to postictal studies in 12 patients firmed the suspected hippocampal sclerosis. All patients underwent temporal lobectomy on the side of the ictal SPECT focus (table 1). Pathology was found in 10 cases (seven showed hippocampal sclerosis, and there were individual cases of ganglioglioma, neocortical gliosis and dysplasia). All but one were seizure free (class 1 of Engel ${ }^{16}$ ) during a mean follow up of 15 (range 4-32) months. Patient 11 had a pronounced improvement in seizure frequency (class 3 of Engel ${ }^{16}$ ), although no pathology was suspected on MRI or found on histological examination.

\section{Discussion}

We analysed the relative blood flow changes in the peri-ictal period as seen in the side to side differences of brain perfusion, by qualitative and quantitative comparisons of cerebral blood flow patterns in the interictal, ictal, and postictal phases. This was done because the determination of absolute CBF by SPECT requires an accurate attenuation correction technique which is not routinely available. It also requires an accurate arterial input function for the tracer, which requires elaborate and time consuming preparations similar to those for PET. Such an approach to blood flow measurement is not currently feasible in the investigation of unpredictable events such as spontaneous seizures.

Ictal scans in all patients showed significant relative global increases in blood flow in the discharging temporal lobe. The SPECT images produced from ${ }^{99 \mathrm{~m}} \mathrm{Tc}-\mathrm{HMPAO}$ injection during or within 30 seconds of the end of the seizure suggests that the ictal temporal hyperperfusion persists beyond the termination of EEG discharge for $60-90$ seconds, as most of the HMPAO that crosses the blood brain barrier does so in the first 60 seconds after the intravenous injection. ${ }^{14}$ This distribution period may be shorter, given the tachycardia that often accompanies seizures. ${ }^{17}$

Injection after this time showed a switch in blood flow patterns, with hypoperfusion of the lateral temporal cortex frequently associated with preserved perfusion or hyperperfusion of the mesial temporal structures. The sequence of changes was thus a significant relative increase in mesial and lateral temporal lobe blood flow with ipsilateral depression of frontal cortex perfusion during the seizure, followed by a reduction in perfusion of the epileptogenic hemisphere with relative preservation of mesial temporal blood flow in the immediate postictal state. Our previous observations ${ }^{11-13}$ suggest that postictal blood flow changes gradually return to normal within 10-30 minutes.

Lee et al noted in two of their ictal studies that cortex surrounding the hyperperfused area was underperfused and suggested that a "steal" phenomenon may be responsible." Alternatively, there may be a depression of neuronal activity in the corical zones adjacent to the disharging temporal lobe and a consequent reduction in the demand for blood flow to those relatively inactive areas. Other ictal studies using SPECT have shown 
increased temporal lobe perfusion, but these did not include observations of frontal and parietal cortex hypoperfusion or the motor strip hyperperfusion observed during hemigeneralisation. ${ }^{5910}$ The motor strip - hyperperfusion found during hemigeneralisation is consistent with the expected haemodynamic response to spread of neuronal discharge to the central region. The agreement of peri-ictal SPECT data with ictal EEG localisation shows the reliability of side and site localisation of SPECT in temporal lobe seizures. The ratio method of quantitation we used confirmed the visual interpretations and allowed a relative measure of the magnitude of changes in CBF.

The hippocampus is the site of seizure generation in most cases of temporal lobe epilepsy. ${ }^{18} 19$ In the present study ictal mesial temporal (hippocampal) hyperperfusion persisted into the postictal period in some cases. This is consistent with the findings previously reported in a larger series of patients, in which depth electrode EEG studies showed total cessation of ictal activity at the time of HMPAO injection. ${ }^{1213}$ Two factors may underlie the ictal and postictal mesial hyperperfusion seen in our SPECT studies. Experimentally, the extracellular cations $\left(\mathrm{H}^{+}\right.$and $\left.\mathrm{K}^{+}\right)$ responsible for the peri-ictal increases in cerebral microflow take some minutes to decline after the end of the seizure. ${ }^{20}$ Secondly, periictal regional $\mathrm{CBF}$ may be coupled to increased non-oxidative glucose metabolism, which reflects hippocampal metabolic activity in physiological neural activation in humans ${ }^{21}$ and experimental hippocampal seizures. ${ }^{22}$

We conclude that the blood flow patterns in temporal lobe epilepsy as shown by SPECT with HMPAO change within a short time frame, with global temporal hyperperfusion switching rapidly (within 90 seconds of seizure end) to hypoperfusion with relative preservation of mesial temporal blood flow. Ictal SPECT images with global temporal hyperperfusion are striking and readily interpretable, whereas the postictal scans show more subtle but characteristic changes that require knowledge of the postictal switch in blood flow. Clinically, therefore, accurate documentation of the time of injection with respect to the video-EEG recording is crucial for proper interpretation. The peri-ictal perfusion patterns provide further evidence that the hippocampus is the generator for most seizures arising in that region. The association of an abnormal synchronised hippocampal electrical discharge with the characteristic rapidly changing blood flow patterns described here may be a pathophysiological hallmark of temporal lobe epilepsy. SPECT has expanded the understanding of pathophysiological events that accompany focal seizures in humans.

The authors gratefully acknowledge the contributions and cooperation they received from all patients, ward staff, and members of the Department of Nuclear Medicine, Austin Hospital, involved in this study. MRN, SFB, and CCR were supported by the National Health and Medical Research Council of Australia.

1 Horsley V. An address on the origin and seat of epileptic disturbance. BMf 1892;i:693-6.

2 Gibbs FA, Lennox WG, Gibbs EL. Cerebral blood flow preceding and accompanying epileptic seizures in man. Arch Neurol Psychiatry 1934;32:257-72.

3 Penfield W. The circulation of the epileptic brain. Ass Res Nerv Dis Proc 1938;18:605-37.

4 Kuhl DE, Engel J Jr, Phelps ME, Selin C. Epileptic patterns of local cerebral metabolism and perfusion in humans determined by emission computed tomography of ${ }^{18} \mathrm{FDG}$ and ${ }^{13} \mathrm{NH}_{3}$. Ann Neurol 1980;8:348-60.

5 Uren RF, Magistretti PL, Royal HD, et al. Single photon emission computed tomography. A method of measunng cerebral blood flow in three dimensions (preliminary cerebral blood flow in three dimensions (preliminary results of studies in patients
Med $\mathcal{F}$ Aust 1983;i:411-3.

6 Engel J Jr, Kuhl DE, Phelps ME. Patterns of human local cerebral glucose metabolism during epileptic seizures. Science 1982;218:64-6.

7 Theodore WH, Newmark ME, Sato SJ, et al. $\left[{ }^{18} \mathrm{~F}\right]$ Fluorodeoxyglucose positron emission tomography in refractory complex partial seizures. Ann Neurol 1983;14: 429-37.

8 Lee BI, Markand ON, Siddiqui AR et al. Single photon emission computed tomography (SPECT) brain imaging using HIPDM: intractable complex partial seizures. Neurology 1986;36:1471-7.

9 Duncan R, Patterson J, Hadley DM, et al. CT, MR and SPECT imaging in temporal lobe epilepsy. $\mathcal{f}$ Neurol Neurosurg Psychiatry 1990;53:11-5.

10 Stefan H, Bauer J, Feistel H, et al. Regional cerebral blood flow during focal seizures of temporal and frontocentral onset. Ann Neurol 1990;27:162-6.

11 Rowe CC, Berkovic SF, Austin M, McKay WJ, Bladin PF. Postictal SPECT in epilepsy. Lancet 1989;i:389-90.

12 Rowe CC, Berkovic SF, Sia STB, et al. Localization of epileptic foci with postictal single photon emission computed tomography. Ann Neurol 1989;26:660-8.

13 Rowe CC, Berkovic SF, Austin MC, McKay WJ, Bladin PF. Patterns of postictal cerebral blood flow in temporal lobe epilepsy qualitative and quantitative analysis. Neurology 1991;41:1096-103.

14 Sharp PF, Smith FW, Gemmell HG, et al. Technetium-99m HMPAO stereoisomers as potential agents for imaging regional cerebral blood flow: human volunteer studies. $\mathcal{f}$ regional cerebral blood fil $1986 ; 27: 171-7$.

15 Jackson GD, Berkovic SF, Tress BM, Kalnins RM, Fabinyi CGA, et al. Hippocampal sclerosis can be reliably detected by magnetic resonance imaging. Neurology 1990 ; 40:1869-75.

16 Engel J Jr. Outcome with respect to seizures. Surgical treatment of the epilepsies. New York: Raven, 1987: 553-71.

17 Blumhardt LD, Smith PEM, Owen L. Electrocardiographic accompaniments of temporal lobe epileptic seizures. Lancet 1986; i: 1051-5.

18 Quesney LF. Clinical and EEG features of complex partial seizures of temporal lobe origin. Epilepsia 1986;27(suppl 2):S27-45.

19 Spencer SS, Williamson PD, Spencer DD, Mattson RH. Human hippocampal seizure spread by depth and subdural recording the hippocampal commissure. Epilepsia 1987;28:479-89.

20 Leniger-Follert E. Mechanisms of regulation of cerebral microflow during bicuculline-induced seizures in anaesthetized cats. F Cereb Blood Flow Metab 1984;4:150-65.

21 Fox PT, Raichle ME, Mintun MA, Dence C. Non-oxidative glucose consumption during focal physiologic neural activity. Science 1988;241:462-4

22 Ackermann RF, Lear JL. Glycolysis-induced discordance between glucose metabolic rates measured with radiolabelled fluorodeoxyglucose andglucose. f Cereb Metab Blood Flow 1989;9:774-85. 